\title{
Re: Urogenital Involvement in the Klippel-Trenaunay-Weber Syndrome. Treatment Options and Results
}

\author{
Fabio C. Vicentini, Francisco T. Denes, Cristiano M. Gomes, Alexandre Danilovic, Frederico \\ A. Silva, Miguel Srougi
}

Division of Urology, University of Sao Paulo School of Medicine (USP), Sao Paulo, Brazil

Int Braz J Urol, 32: 697-704, 2006

To the Editor:

With the intention to provide more information about this rare and difficult disease, we communicate that the boy from the Case 3 underwent a right transfemoral amputation due to massive enlargement and walking inability in November 2006. In January 2007, he had a massive enterorrhagia probably related to the growth of the vascular tumor and involvement of the colon. Despite multiple transfusions and medical measures, stabilization was not achieved and he died before surgical procedure could be attempted. The patient of the Case 1 is a mulatto man, and not a black man as described.
We wish to thank Dr. José Luis Borges de Mesquita who participated in the treatment of Case 5 and Dr. Antonio Marmo Lucon, who provided the data of the patient of the Case 1 .

Dr. Fabio C. Vicentini Division of Urology, School of Medicine, University of Sao Paulo, USP Sao Paulo, SP, Brazil Email:fabio.vicentini@sbu.org.br

\section{Re: Pudendal Nerve Latency Time in Normal Women via Intravaginal Stimulation}

\author{
Geraldo A. Cavalcanti, Gilberto M. Manzano, Lydia M. Giuliano, Joao A. Nobrega, \\ Miguel Srougi, Homero Bruschini
}

Departments of Urology and Neurology, Federal University of Sao Paulo, UNIFESP, Paulista School of Medicine, Sao Paulo, SP, Brazil

Int Braz J Urol, 32: 705-712, 2006

To the Editor:

We have to congratulate Cavalcanti et al. for their very nice study adding information on pudendal pathologies. They concluded that the vaginal approach represents an alternative for pudendal nerve distal 
motor latency time, with similar results to those achieved through the transrectal approach. Normative values obtained might serve as a comparative basis for subsequent physiopathological studies.

We have for long time used the 2 approaches (vaginal and rectal) and find sometimes bizarre results. We recall that for measuring pudendal nerve motor time conduction a special device was developed at the St Mark's London Hospital. It consists of a bipolar stimulating electrode fixed on a gloved index finger. A pair of surface recording electrodes is placed $3 \mathrm{~cm}$ proximally on the base of the finger. Using a rectal pathway the stimulating electrode is placed near the ischial spine. The recording electrode is at the level of the anal sphincter. Geraldo and al. describe a transvaginal stimulation. Pudendal nerve motor latency time obtained by transrectal or transvaginal stimulation has to be viewed with some uncertainty and doubts because we must have in mind the imprecision of the stimulation point, which is at the level of the ischial spine.

An entrapment at this site cannot be detected and distortion of the stimulating potential by the different layers of tissues to go through can explain normal results even in presence of a neuropathy (Cavalcanti et al.). For the last 6 months, we have been use an insulated regional analgesia needle with

\section{REPLY BY THE AUTHORS}

We are grateful for the interest shown in our study and especially thank you for information on potentials improvements in the technique used by Dr. Eric de Bisschop and Dr. Jean Pierre Spinosa.

They shared their experience in obtaining the pudendal nerve terminal motor latency (PNTML) for both approaches (vaginal and rectal). We agree that imprecision of the stimulation point and the interposition of different layers of tissues between the pudendal nerve and the stimulus electrodes represent technical and biological factors that may interfere in the M-wave recordings, even reaching the electro-neuro-stimulation port (100 or $120 \mathrm{~mm}$ ) placed above the ischial spine (by a transmuscular perineal route parallel to the ischiorectal fossa) to stimulate the pudendal nerve. The recording is made with a circular bipolar electrode placed in the anal sphincter with some advantages: the stimulations are made above the ischial spine permitting a detection of an entrapment even at this site, it localize with accuracy the site of entrapment (sacrospinous ligament, falciformis process, pudendal tunnel), there is less or no distortion of the potential, it can be used in women and men, it can be used as an intraoperative monitoring, it inform the surgeon at the time of the decompression on the ongoing of the procedure or the necessity of completing it, therefore, improving the surgical procedures.

\section{Dr. Eric de Bisschop \\ Clinique de Montchoisi \\ Lausanne, Switzerland \\ E-mail: rajeshree@free.fr}

Dr. Jean Pierre Spinosa

Department of Gynecology, Hôpital de Morges

Morges, Switzerland

E-mail:spinosa@deckpoint.ch

supramaximum stimulus. However, the latency value must not be altered by these factors.

They also described an interesting and novel technique to obtain the PNTML by utilization of needle electrode for pudendal nerve stimulation instead of St. Mark's electrode. Other authors also designed an intra-rectal incurvated metallic rod stimulator with similar arguments, as an alternative method to assess the PNTML (1). A needle stimulator could obtain more quality recordings because the direct stimuli of the nerve can be reached and specific stimulation areas can be localized with accuracy. But the 
St. Mark's electrode used in the study has the distance between the stimuli and recording sites known in a region of difficult access for measuring. This would become the method more standardized and appropriate for pelvic floor, besides probably causing less discomfort than the transmuscular perineal route. These alternative methods for PNTML assessment might be useful in routine practice, mainly for intraoperative monitoring. Nevertheless it should be tested in further studies.

\section{Reference}

1. Lefaucheur JP, Yiou R, Thomas C: Pudendal nerve terminal motor latency: age effects and technical considerations. Clin Neurophysiol. 2001; 112: 4726.

\title{
Re: Percutaneous Nephrolithotomy with and without Retrograde Pyelography: A Randomized Clinical Trial
}

\author{
Ali Tabibi, Hamed Akhavizadegan, Kia Nouri-Mahdavi, Mohammad Najafi- Semnani, \\ Mojgan Karbakhsh, Ali R. Niroomand
}

Section of Urology, Dr Labbafinejad Hospital, Shahid Beheshti University of Medical Sciences, Tehran, Iran, Section of Urology, Baharloo Hospital (HA) and Department of Community Medicine (MK), School of Medicine, University of Tehran, Tehran, Iran

Int Braz J Urol, 33: 19-24, 2007

To the Editor:

This article by Tabibi et al. evaluates whether there is a benefit to performing cystoscopic ureteral catheter placement, for retrograde opacification of the renal pelvis and calyces, in order to obtain access prior to percutaneous nephrolithotomy (PCNL). The study was randomized to either no catheter placement (intervention group) or catheter placement (control group). No statistical differences were found when comparing demographic or intraoperative parameters (surgical duration, radiation exposure, hospital stay, postoperative fever) between the 2 groups. Interestingly, postoperative hemoglobin decrease was found to be significantly higher in the catheterized group ( $\mathrm{p}<0.001)$. While no statistical difference was identified for outcome $(\mathrm{p}=0.136)$, a greater percentage of patients were found to be "stone free" on KUB in the catheterized group than the noncatheterized group, $93 \%$ vs. $79 \%$, respectively. Based on these results, it would appear that while retrograde placement of a ureteral catheter does not influence the safety or duration of the procedure, it may facilitate the efficacy of PCNL in terms of achieving stone free status.

The decision for selecting a noncatheterized versus a catheterized approach should take into account the experience of the individual obtaining access as well as the clinical scenario in terms of stone location and burden. In many institutions, initial access is obtained by an experienced interventional radiologist followed by PCNL that is performed by the urologist. Even in this circumstance, it is not uncommon for the interventionalist to request a ureteral catheter for retrograde opacification of the 